\title{
Identification de quelques plantes utilisées en médecine ethnovétérinaire à Sinématiali (Nord de la Côte d'Ivoire)
}

\author{
${ }^{*}$ KONE Kéassemon Hervé Cédessia', COULIBALY KiyinIma', KONAN Kouakou Severin² \\ 1Département de Biologie Végétale, UFR des Sciences Biologiques, Université Péléfero Gon Coulibaly BP 1328 \\ Korhogo \\ 2Département Biochimie-Génétique, UFR des Sciences Biologiques, Université Pelefero Gon Coulibaly BP 1328 \\ Korhogo \\ * Auteur correspondant : Email : hervekone2001@gmail.com \\ Original submitted in on $6^{\text {th }}$ December 2018. Published online at www.m.elewa.org/journals/ on $31^{\text {st }}$ March 2019 \\ https://dx.doi.org/10.4314/jab.v135i1.3
}

\section{RESUME}

Objectif: Contribuer à la préservation et à une meilleure connaissance des plantes utilisées pour le traitement des pathologies bovines dans le département de Sinématiali

Méthodologie et résultats: Des enquêtes ethnobotaniques ont été réalisées à l'aide de fiches de questionnement auprès de 40 éleveurs adultes et expérimentés du département. Ces enquêtes ont permis d'inventorier 24 espèces végétales réparties en 24 genres et 18 familles botaniques. Ces espèces entrent dans la composition de 25 recettes de traitement thérapeutique de divers maux bovins dont les plus fréquents sont la diarrhée, le mal de ventre, les vers intestinaux et le paludisme. Les espèces entrant dans les diverses compositions appartiennent majoritairement à la famille botanique des Caesalpiniaceae. Les feuilles sont les organes les plus utilisées (52\%). La forme d'utilisation de ces plantes est la décoction (72\%).

Conclusion et application des résultats : Ce travail constitue une étude préliminaire devant servir de base à des études et analyses pharmacologiques plus approfondies des plantes recensées.

Mots-clés : Ethnobotanique, Ethnovétérinaire, plantes médicinales, thérapeute, Sinématiali, Côte d'Ivoire.

\begin{abstract}
Objective: Contribute to the preservation and better knowledge of plants used for the treatment of bovine diseases in the department of Sinematiali.

Methodology and results: Ethnobotanical surveys were conducted using questionnaires from 40 adult and experienced breeders in the department. These surveys allowed inventorying 24 plant species divided into 24 genera and 18 botanical families. These species form part of recipes for the therapeutic treatment of various cattle ailments, the most frequent of which are diarrhea, stomach ache, intestinal worms and malaria. The species entering the various compositions belong mainly to the botanical family of Caesalpiniaceae. Leaves are the most used organs (52\%). The most common form of use of these plants is decoction (72\%).

Conclusion and application of results: This work is a preliminary study to serve as a basis for further pharmacological studies and analyzes of identified plants.
\end{abstract}

Keywords: Ethnobotany, Ethnoveterinary, medicinal plants, therapy, Sinématiali, Côte d'Ivoire 


\section{INTRODUCTION}

Le développement de l'élevage et l'augmentation de la production animale est l'enjeu majeur de la politique nationale d'investissement agricole en Côte d'lvoire. Cependant, le pays se trouve toujours fortement dépendant de l'extérieur dans ses approvisionnements en viande. Selon le ministère des ressources animales et halieutiques, pour la seule année 1994, le gouvernement ivoirien a dépensé plus de 60 Milliards pour approvisionner sa population en protéines animales. Cet élevage est confronté à de nombreuses maladies dont la plupart sont des problèmes gastriques et maladies parasitaires. Les pertes causées par les nématodes et les parasites gastro-intestinaux sont considérables (Anonyme 1, 1995). Elles sont les principales causes de mortalité et d'importantes pertes économiques dans les systèmes d'élevage bovin et ovin. Par ailleurs, le manque d'encadrement sanitaire adéquat, l'insuffisance de fourrage, la transhumance en saison sèche et les conflits récurrents entre éleveurs et agriculteurs concourent également à la réduction de la production. Pour remédier à cette situation, les populations du nord en général et ceux du département de Sinématiali en particulier, font recours à la médecine traditionnelle. En effet, les plantes médicinales constituent le moyen le plus utilisé en milieu rural pour résoudre les problèmes de santé publique. Selon l'OMS (2002), plus de $80 \%$ de la population africaine a recours aux plantes pour ses besoins en soins de santé. En médecine vétérinaire aussi, la phytothérapie est largement sollicitée par les éleveurs traditionnels. La médecine ethno-vétérinaire (MEV) est un terme scientifique pour les soins de santé animale

\section{MATERIEL ET METHODES}

Milieu d'étude: L'enquête a été menée dans le département de Sinématiali, dans la région du Poro au nord de la Côte d'Ivoire. Sinématiali se situe à $27 \mathrm{~km}$ de Korhogo et à environ $600 \mathrm{~km}$ d'Abidjan. C'est est une zone propice à l'élevage des bovins et ovins. Avec un faible pouvoir d'achat, la population est majoritairement pauvre. Vue la facilité à préparer et à administrer les médicaments à base de plantes et sa gratuité, la traditionnelle qui englobe les connaissances, compétences, les méthodes, pratiques et croyances concernant les animaux visant au seins des communautés (McCorkle, 1986 in Merhazi et al.). Compte tenu de la facilité à préparer et à administrer les médicaments à base de plantes et sa gratuité, la médecine ethno-vétérinaire occupe une place importante dans les pratiques des éleveurs des pays en voie de développement (Merazi et al., 2016). En Côte d'ivoire, des travaux ont montré l'intérêt de l'usage des plantes en santé animale (Aké Assi et Guinko, 1991 ; Koné et Kamanzi Atindehou, 2008). Ces travaux ont montré que la flore ivoirienne possède des espèces végétales susceptibles de jouer un rôle dans le contrôle des pathologies bovines et ovines. Notons qu'il n'existe pas de service vétérinaire dans le département de Sinématiali, ce qui cause un véritable problème pour le traitement des animaux. Pour pallier à ce problème, les éleveurs de la localité ont recours aux plantes médicinales. II apparaît donc urgent d'identifier ces plantes. Afin d'empêcher nos remèdes de disparaitre avec leur plantes et avec les personnes âgées, et dans le but d'améliorer les informations sur les applications thérapeutiques et traditionnelles en médecine vétérinaire et assurer un lien entre les pratiques ancestrales et la médecine conventionnelle, une enquête sur terrain auprès des éleveurs traditionnels de bovins a été réalisée. Cette enquête qui avait pour objectif l'identification de quelques plantes à usage médicinale pour des systèmes d'élevages bovin a été réalisée dans le département de Sinématiali au nord de la Côte d'Ivoire.

médecine ethno-vétérinaire occupe une place non négligeable dans les pratiques des éleveurs dans la région du Poro et en particulier ceux de Sinématiali. La principale langue parlée en ville et dans les villages environnant est le Nanfara (figure 1).

Matériel végétal : Ce travail a porté sur les espèces du règne végétal en général et en particulier sur les plantes présentant un intérêt thérapeutique pour les 
systèmes d'élevage bovin. Une centaine de parcs de bovin et d'ovin ont été visités au cours de cette enquête. Deux(2) variétés de bovin selon l'absence ou la présence de Bosse ont été rencontrées. II s'agit des zébus avec boss (Bos indicus) et des taurins sans boss (Bos taurus). Les Taurins regroupent les races N'dama, Baoulé et Lagunaire. Notons que la race Baoulé est la plus rencontrée dans Le département de Sinématiali, les autres y sont en voie d'extinction. La race zébune rencontrée est le Zébu peulh soudanais. On rencontre également divers métis issus $d u$ croisement entre mâles zébus et femelles Baoulés.
Enquêtes ethno-vétérinaires : Au cours de l'enquête, 40 guérisseurs et éleveurs de bovins et ovins matures et expérimentés ont été visités et questionnés. Les informations collectées concernent les plantes utilisées pour traiter les maladies fréquemment contractées par les bêtes. Les noms locaux, les organes ou parties de la plante utilisées, les indications thérapeutiques, les moments de récolte et les modes d'administration de ces plantes médicinales ont été répertoriés.

Traitement des données : Les données recueillies ont été saisies avec le logiciel Word, analysées et traitées à l'aide de Microsoft Excel 2007. 


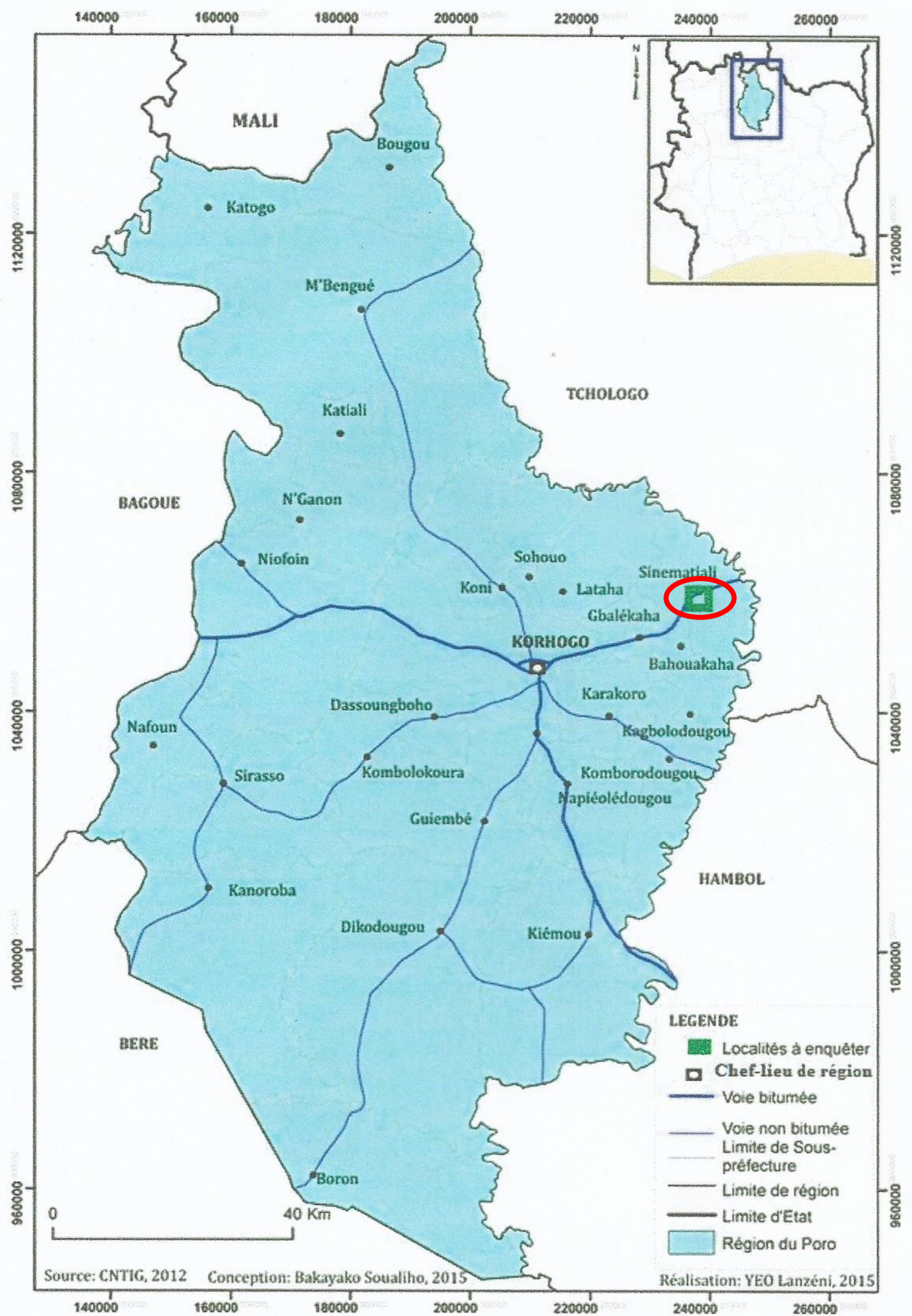

Figure 1 : Carte de la région du Poro (Côte d'Ivoire) 


\section{RESULTATS}

Pathologies des bovins: Dans le département de Sinématiali, 13 maladies dont les plus fréquemment rencontrées sont la diarrhée $(67,5 \%)$, les maux de ventre, et les zoonoses à Plasmodium spp. chez les bovins. A cela s'ajoutent les maladies parasitaires telles que les tiques et les vers intestinaux (figure 2).

Plantes médicinales utilisées: Pour les traitements des pathologies bovines, l'enquête a permis de recenser 24 espèces appartenant à 24 genres répartis entre 18 familles botaniques (Tableaux 1 à 7 ). La famille la plus représentée est celle des Caesalpiniaceae $(22,22 \%)$ dont les espèces sont utilisées pour traiter majoritairement la diarrhée (Tableau 1). Les espèces les plus utilisées de cette famille sont Detarium microcarpum et Pilostigma thonnigii. En second plan, viennent les Euphorbiaceae, Caricaceae, Rubiaceae, Anacardiaceae, Meliaceae et les Combretaceae avec $11,11 \%$ chacune. Les plantes utilisées pour le traitement des vers intestinaux sont Trema guineensis, Cassia siamea et Carica papaya (Tableau 2). Pour le traitement des douleurs abdominales chez les bovins, recours est fait à Securidaca longipedunculata, Alternanthera pungens, Annona senegalensis, Diopyros mespiliformis, Mitragyna inermis, Trichilia emetica et Sclerocarya birrea (Tableau 3). Les espèces Carica papaya, Guiera senegalensis, Anthostema senegalense et Ficus vallischoudae sont utilisées pour le traitement les zonose à Plasmodium spp. (Tableau 4).Le traitement des tiques est aussi une préoccupation pour les éleveurs du département. IIs ont utilisent pour ce fait Waltheria indica et Trichilia emetica (Tableau 5) .En fin, dans les tableaux 6 et 7 sont listées les espèces utilisées par les éleveurs de Sinématiali pour traiter la toux et les fractures chez les bovins. Ce sont Crossopteryx febrifuga et Anogeissus leiocarpus pour la toux et Lannea barteri pour les fractures.

Parties des plantes utilisées: Les remèdes sont confectionnés le plus fréquemment à partir des feuilles $(45,83 \%)$, des racines $(16,66 \%)$, des écorces de tronc $(12,5 \%)$. Les autres parties utilisées représentent 25,01\% (Tableau 8).

\section{Fréquence des pathologies bovines (\%)}

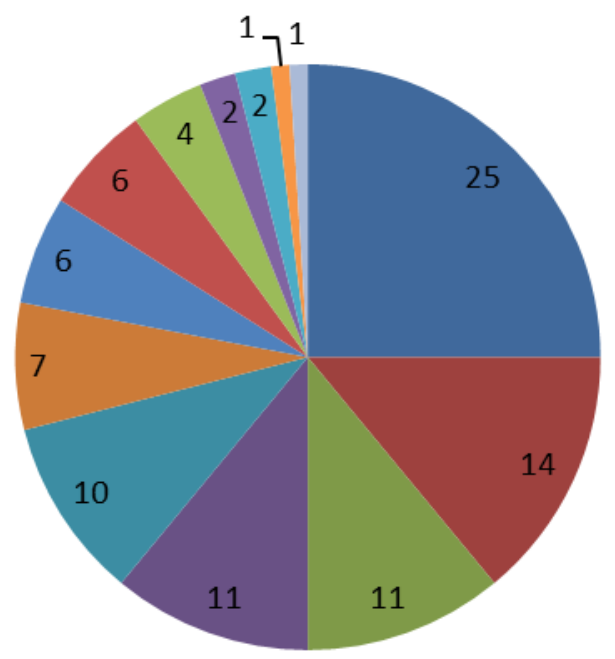

Figure 2 : pathologies les plus fréquemment rencontrées en élevage de bovins 

à Sinématiali (Nord de la Côte d'Ivoire)

Tableau 1 : Plantes utilisées pour traiter la Diarrhée chez les bovins

\begin{tabular}{l|l|l|l}
\hline Espèces & Parties utilisées & Familles & Nom local \\
\hline Sida acuta & Feuilles & Malvaceae & Tchegbènèbalier \\
Euphorbia hirta & Plante entière & Euphorbiaceae & Goriyirimin \\
Althernanthera pungens & Plante entière & Amaranthaceae & Kabelisaharga \\
Detarium microcarpum & Feuilles & Caesalpiniaceae & Gbogbo \\
Piliostigma thonnigii & Feuilles & Caesalpiniaceae & Olofoo \\
Pericopsis laxiflora & Tiges & Fabaceae & Kolo \\
Psidium guajava & Feuilles & Myrtaceae & Goyavé \\
Adansonia digitata & Ecorce du tronc & Bombacaceae & Zihintegué \\
\hline
\end{tabular}

Tableau 2 : Plantes utilisées pour traiter les vers intestinaux chez les bovins

\begin{tabular}{l|l|l|l}
\hline Espèces & Parties utilisées & Familles & Nom local \\
\hline Trema guineensis & Feuilles & Ulmaceae & Mou \\
Cassia siamea & Feuilles & Caesalpiniaceae & Cassia \\
Carica papaya & Graines séchées & Caricaceae & Mahandji \\
\hline
\end{tabular}

Tableau 3 : Plantes utilisées pour traiter les douleurs abdominales chez les bovins

\begin{tabular}{l|l|l|l}
\hline Espèces & Parties utilisées & Familles & Nom local \\
\hline Securidaca longipedunculata & Racines & Polygalaceae & Felimin \\
Alternanthera pungens & Plante entière & Amaranthaceae & Kabelisaharga \\
Annona senegalensis & Feuilles & Annonaceae & Namourgo \\
Diopyros mespiliformis & Feuilles & Ebenaceae & Gnantchou \\
Mitragyna inermis & Feuilles & Rubiaceae & Liyirimin \\
Trichilia emetica & feuilles-racines & Meliaceae & Gotounonssouko \\
Sclerocarya birrea & feuilles-racines & Anacardiaceae & Sanhanlogo \\
\hline
\end{tabular}

Tableau 4 : Plantes utilisées pour traiter la zoonose à Plasmodium spp. chez les bovins

\begin{tabular}{l|l|l|l}
\hline Espèces & Parties utilisées & Familles & Nom local \\
\hline Carica papaya & Feuilles & Caricaceae & Mahandji \\
Guiera senegalensis & Ecorce du tronc & Combretaceae & Koungbè \\
Anthostema senegalense & Feuilles & Euphorbiaceae & Laconemon \\
Ficus vallis-choudae & Ecorce du tronc & Moraceae & Yibbé \\
\hline
\end{tabular}

Tableau 5 : Plantes utilisées pour traiter les tiques chez les bovins

\begin{tabular}{l|l|l|l}
\hline Espèces & Parties utilisées & Familles & Nom local \\
\hline Waltheria indica & Racines & Sterculiaceae & wôfigué, koungbè \\
Trichilia emetica & Racines & Meliaceae & Gotounonssoukro \\
\hline
\end{tabular}

Tableau 6 : Plantes utilisées pour traiter la toux chez les bovins

\begin{tabular}{l|l|l|l}
\hline Espèces & Parties utilisées & Familles & Nom local \\
\hline Crossopteryx febrifuga & Feuilles & Rubiaceae & Quiquina \\
Anogeissus leiocarpus & Feuilles & Combretaceae & N'galama \\
\hline
\end{tabular}

Tableau 7 : Plantes utilisées pour traiter les fractures toux chez les bovins

\begin{tabular}{l|l|l|l}
\hline Espèces & Parties utilisées & Familles & Nom local \\
\hline Lannea barteri & Racines & Anacardiaceae & Faru \\
\hline
\end{tabular}


Plantes et indications thérapeutiques: Ces organes végétaux frais ou $\mathrm{sec}$, provenant d'une plante ou d'une association de plantes, sont utilisés majoritairement sous forme de décoctions (72\%). Les ingrédients tels que le sel, le citron, le gingembre, et le miel entrent dans la confection de certaines recettes. Les remèdes sont administrés par voie orale dans $92 \%$ des cas (Tableau 8). Pour les plantes identifiées, les guérisseurs et les éleveurs n'ont pas mentionné des effets secondaires.

Tableau 8 : Plantes, organes utilisés et indication thérapeutique

\begin{tabular}{|c|c|c|c|}
\hline Nom local & Nom binomial & Organe utilisé & Mode d'administration \\
\hline Yibbé & Ficus vallis-choudae & Ecorce & Décoction, un verre, 2 fois par jour \\
\hline Goyavé & Psidium gujava & Feuilles & Décoction, un verre, 2 fois par jour \\
\hline Zihintigué & Adansonia digitata & Ecorce & Décoction, un verre, 2 fois par jour \\
\hline Dihin & Lannea acida & Feuilles/racines & Décoction, un verre, 2 fois par jour \\
\hline Palcthoi & Detarium senegalense & Ecorce & Décoction, un verre, 2 fois par jour \\
\hline Tipkèri & Hymenocardia acida & Racines & Décoction, un verre, 2 fois par jour \\
\hline Guenmin & Anogeissus leiocarpus & Ecorce & Décoction, un verre, 2 fois par jour \\
\hline Kounbgè & Guiera senegalensis & Feuilles/écorces & Décoction, un verre, 2 fois par jour \\
\hline Guenmin & Anogeissus leiocarpus & Feuilles/tiges/racines & Décoction, un verre, 2 fois par jour \\
\hline Nèrè & Parkia biglobosa & Ecorce & Décoction, un verre, 2 fois par jour \\
\hline Lirimin & Mitragyna inermis & Feuilles & Décoction, un verre, 2 fois par jour \\
\hline Gnantchou, & Diospyros mespiliformis & Feuilles & Décoction, un verre, 2 fois par jour \\
\hline Kuntango & Sclerocarya birrea & Feuilles/racines & Décoction, un verre, 2 fois par jour \\
\hline Yiriwaha & Entada africana & Racines & Décoction, un verre, 2 fois par jour \\
\hline Sidjènefielguè & Ficus glomosa & Feuilles & Décoction, un verre, 2 fois par jour \\
\hline Gnantchou & Diospyros mespiliformis & Feuilles & Décoction, un verre, 2 fois par jour \\
\hline Souhonmon & Lophira lanceolata & Racines & Décoction, un verre, 2 fois par jour \\
\hline Sayiri & $\begin{array}{l}\text { Securidaca } \\
\text { longipedunculata }\end{array}$ & Racines & Décoction, nettoyer la plaie \\
\hline Gotounonssoukro & Trichilia emetica & Feuilles & Poudre délayée dans de l'eau chaude \\
\hline Mahandji & Carica papaya & Graines & Poudre délayée dans de l'eau chaude \\
\hline Mandja & Cyperus articulatus & Tubercules & Poudre délayée dans de l'eau chaude \\
\hline Kounbgè & Guiera senegalensis & Ecorces & poudre dans l'eau chaude, 2 fois/jour \\
\hline Yibbé & Ficus vallis-choudae & Feuilles & macérer avec sel, répandre sur la partie \\
\hline Mahandji & Carica papaya & Feuilles & Macérât, boisson \\
\hline Mahandji & Carica papaya & Feuilles & Macérât avec citron, boisson \\
\hline
\end{tabular}

\section{DISCUSSION}

Cette enquête ethnobotanique auprès de 40 éleveurs du département de Sinématiali a permis de recenser puis d'identifier au total 24 plantes médicinales reparties en 24 genres appartenant à 18 familles différentes. La population de Sinématiali utilise plus ou moins les même plantes pour le traitement des pathologies bovines et ovines. La différence réside dans le mode d'administration des remèdes en tenant compte du poids de l'animal à traiter. Parmi ces plantes, l'utilisation de Carica papaya (Caricaceae) pour le traitement du paludisme, des vers intestinaux semble la plus répandue. En effet, le papayer possède de nombreuses vertus médicinales. En phytothérapie humaine, on utilise aussi bien le fruit que les feuilles, les graines, le latex ou les racines. Selon la partie utilisée, le papayer est un purgatif, un antiinflammatoire ou un agent ayant des effets positifs sur la digestion (Anonyme 2 ; Mpoame et Essomba, 2000). En nous référant aux travaux de Sangaré (2015), ces plantes ont été aussi rapportées comme des plantes médicinales utilisées ailleurs dans la région du Poro. A Ferkessédougou, pour réduire l'incidence des pathologies bovines et ovines, la population utilise couramment les espèces appartenant à la famille des Meliaceae $(15,15 \%)$ et celle des Caesalpiniaceae $(12,12 \%)$. Les maladies fréquemment traitées y sont la diarrhée et les vers intestinaux. En outre, Carica papaya (Caricaceae), Ficus vallis-choudae (Moraceae), Anogeissus leiocarpus (Combretaceae) et Detarium microcarpum (Caesalpiniaceae) ont été recensées 
comme remèdes dans le traitement respectif du paludisme, des tiques, et de la diarrhée dans les systèmes d'élevages bovin et ovin à Korhogo (N'gouan, 2015). Pour quelques espèces végétales très utilisées traditionnellement, un lien a été établi entre les indications thérapeutiques et les propriétés déjà montrées dans des travaux antérieurs. C'est le cas d'Anogeissus leiocarpus (Combretaceae) qui est utilisée pour traiter la diarrhée. L'activité anti diarrhéique de cette plante serait due à la forte teneur en tannin et à la présence de la quercétine (Neuwinger, 1994). A travers toute l'Afrique, pour combattre les vers intestinaux et les désordres Gastro-intestinaux, les populations ont recours à Annona senegalensis de la famille des Annonaceae. Les études menées sur les propriétés chimiques et pharmacologiques de la plante révèlent que l'acide kaurénoïque est responsable de son efficacité sur les maladies, mais cette espèce présente une très forte toxicité selon Neuwinger (1994). En Côte d'Ivoire comme ailleurs en Afrique, Securidaca longepedunculata (Polygalaceae) est très utilisée pour traiter divers problèmes de santé. Cette plante, d'un si grand intérêt thérapeutique, est hélas toxique. La sécurinine isolée dans les écorces de tronc et les saponines dans les racines seraient responsables de la toxicité de la plante (Neuwinger, 1994). Toutefois, si les écorces de racines sont utilisées sous forme d'un macéré aqueux à $10 \%$, la toxicité aiguë serait pratiquement nulle. Ainsi, Lejoly et al. (1994) conseillent

\section{CONCLUSION}

Ce travail a permis de recenser puis d'identifier quelques plantes médicinales pour les systèmes d'élevages bovins et ovins dans le département de Sinématiali. II a montré que la flore de ce département renferme encore un riche potentiel de plantes susceptibles de jouer un rôle dans le traitement de diverses maladies. Les plantes couramment utilisées dans le traitement de diverses infections animales sont Sclerocarya birrea (Anacardiaceae), Carica papaya (Caricaceae), Anogeissus leiocarpus (Combretaceae), Cassia siamea (Caesalpiniaceae), et Parkia biglobosa

\section{REFERENCES BIBLIOGRAPHIQUES}

Aké-Assi L et Guinko S., 1991. Plantes utilisées en médecine traditionnelle en Afrique de l'ouest. Edition. Roche, Switzerland, 151 pages.

Anonyme 1, 1995. Rapport d'activités du MRAH (Ministère des Ressources Animales et Halieutiques). Document interne. 56 pages. l'administration de cette plante comme remède que si le dosage est fait sous un contrôle strict. On note que le nombre total d'espèces végétales identifiées est relativement faible sur une surface aussi grande que le département de Sinématiali. Plusieurs raisons peuvent expliquer cette observation. Nous pouvons incriminer en premier lieu certaines pratiques culturales qui consistent au défrichement de nouvelles terres pour la mise en place de cultures exigeantes telles l'igname. A cela s'ajoute la coupe intense de bois de chauffe pour la cuisson du "Tchapalo ", boisson très appréciée dans la région du Poro. Les coupes concernent de façon générale les jeunes plantes. La faible richesse peut s'expliquer aussi par les feux de brousses, la saison sèche très rude, le changement climatique et la surexploitation ou la surutilisation. Par ailleurs, les mauvaises techniques de récolte des plantes peuvent aussi être à l'origine de la disparition ou de la raréfaction de certaines d'entre elles telles que Sclerocarya birrea (Anacardiaceae), Ficus vallischoudae (Moraceae) et Annona senegalensis (Annonaceae). Les plantes sont le plus souvent déterrées pour la récolte des racines utilisées pour la confection des remèdes traditionnelles. Aussi, insistons-nous sur le fait que si rien n'est fait pour protéger la biodiversité, les plantes à usage médicinales pour l'homme et les animaux disparaitront d'ici quelques décennies dans le département voire dans la région du Poro.

(Mimosaceae). L'étude a aussi montré la rareté de Sclerocarya birrea (Anacardiaceae) du fait de sa forte utilisation. Cette plante est en voie d'extinction dans le nord du pays. Des campagnes de sensibilisation doivent être organisées afin de sensibiliser les paysans et les encourager à étendre leurs pratiques de conservation à un plus grand nombre de plantes utiles ou à les domestiquer. Ceci permettrait une bonne dynamique des plantes médicinales et limiterait la menace de disparition qui frappe déjà certaines d'entre elles.

Anonyme 2, 2018. Doctissimo: www.doctissimo.fr /html/sante/phytothérapie/plante-médicinale/ papayer.htm

Koné M. et Kamanzi Atindehou K., 2006. Inventaire Ethnomédical Evaluation de l'Activité Anthelminthique des Plantes médicinales 
utilisées en Côte d'Ivoire. Pham. Méd.Trad. Afr. Vol. X/V: pp. 55-72.

Lejoly J., Richel T., Van Essche K. 1994. Plantes Africaines utilisées en médecine traditionnelle. Proceeding of the XIllth Plenary Meeting AETFAT, Malawi, 1: 197-217

McCorkle C. M., 1986. An introduction to ethnoveterinary research and development: Publisher not identified.

Merazi Y., Hammadi K., Fedoul F., 2016. Approche ethno-vétérinaire des plantes médicinales utilisées dans la région de Sidi Bel AbbesAlgérie. European Scientific Journal. Edition 2016 Vol 12. No 18 ISSN 1857-7881.

Mpoame M. et Essomba L. I. (2000). Essai de traitement contre les parasitoses gastrointestinales du poulet avec des décoctions aqueuses de graines de papaye (Carica papaya). RevElevMédVét Pays Trop, 53: 2325.

N'gouan, 2015. Identification de quelques plantes à usage médicinale pour système d'élevage bovin et ovin à Ferkessédougou (Nord de la Côte d'Ivoire), Mémoire de Licence. UFR des sciences biologiques, Université Pelefero Gon Coulibaly. X pages.

Nuewinger H. D., 1996. African Ethnobotany Poisons and Drugs Chemistry PharmacologyToxicology. Edition Chapman \& Hall, London. Glasgow. Weinheim. Tokyo. Melbume. Madras, $941 \mathrm{p}$.

OMS, 2002. Stratégie de l'OMS (Organisation Mondiale de la Santé), pour la médecine traditionnelle (2002-2005).WHO/EDM/TKM/ 2002 Genève. 